\title{
Modelling the influence of strain and strain rate on the thermal softening during dynamic loading of ductile metals
}

\author{
Giuseppe Mirone* and Raffaele Barbagallo \\ University of Catania, DICAR - Department of Civil Engineering and Architecture, Via Santa Sofia 64 \\ - 95123 - Catania, Italy.
}

\begin{abstract}
Although the combined effect of strain rate and temperature on the behaviour of metals is widely recognized, no universally accepted viewpoints are available about the physical phenomena. Experiments on a highly ductile A2-70 steel, performed at moderate dynamic rates $\left(10 \mathrm{~s}^{-1}\right)$ and different initial temperatures $\left(20\right.$ to $\left.150{ }^{\circ} \mathrm{C}\right)$, are firstly aimed here at assessing whether the thermal softening previously verified at static rates on the same steel is also suitable for describing now the mixed effect of dynamic rates and consequent variable temperatures, or further contributions to the thermal softening are necessary for describing such mixed effects. A general multiplicative model of the dynamic hardening is proposed, based on a static flow curve at room temperature to be increased by the dynamic amplification and to be decreased by the thermal softening, the latter incorporating the known "static component" depending on both strain and constant temperatures, together with a new "dynamic component" incorporating the dependence on the temperature variation and promoted by fast straining. The dynamic amplification of the stress is then obtained from another series of dynamic tests ran at initial room temperature and four nominal strain rates between 1 and $1800 \mathrm{~s}^{-1}$. The trend obtained is compatible with the seizing of the strain rate effect beyond necking onset, already found for other metals in previous works. All the experiments are based on the acquisition of the current load (by load cells for the testing machine and by strain gauges for the Hopkinson bar) and of the current cross section through optical diameter measurements by a fast camera; then, the effective current values of true stress-true strain-true strain rate are measured on a semi-local basis over the neck section at different instants during the test.
\end{abstract}

\section{Introduction}

The characterization and modelling of the behaviour of metals at high temperatures and high strain rates have been extensively studied in the literature, but there are still open questions regarding the combined effects of strain, strain rate and temperature. In order to correctly identify the above combined effects, the equivalent stress-strain curves of the material at different strain rates and temperatures must be evaluated. Regarding this aspect, Peroni et al. [1] developed a neck-profile-based inverse method while Sasso et al. [2] proposed a novel

\footnotetext{
* Corresponding author: gmirone@ dii.unict.it
} 
procedure for the FEM-based calibration of material curves. The equivalent curve can also be obtained from the true curve without numerical iterations, e.g., by the Bridgman [3] method or by the more recent and simpler MLR method proposed by Mirone [4]. The experimental true curve necessary for implementing such two methods can be obtained, for cylindrical specimens, by means of optical measurements of the diameter during the test or, for rectangular specimens, through a dedicated material-independent method able to deliver the true variables from the engineering ones as in Mirone et al. [5].

In dynamic tests, the material temperature spontaneously increases due to the conversion of plastic work into internal heat under almost adiabatic exchange conditions. Subsequently, in order to evaluate the stress amplification due to the strain rate, the thermal softening acting under dynamic conditions must be identified within the experimental data and isolated from the dynamic stress amplification. The material temperature evaluation in dynamic tests involves the Taylor-Quinney coefficient. In Kapoor \& Nemat-Nasser [6] and Walley et al. [7], it is demonstrated that the plastic work is almost completely converted into heat, while Jovic et al. [8] and Rittel et al. [9] calculated that lower fractions of plastic work can be converted into heat for the same material depending on the stress state conditions.

Another relevant aspect of the dynamic hardening is the interaction between temperature, strain rate and plastic instability. The authors previously found in [10][11][12][13] that the strain rate rapidly increases at necking onset due to the progressing strain localization, while the strain rate sensitivity quits evolving and remains constant up to failure. Zhang et al. [14] and Zhang et al. [15] analysed the tensile response and strain localization of a near $\alpha \mathrm{Ti} 3 \mathrm{Al} 2.5 \mathrm{~V}$ at quasi-static, medium and high strain rates at room and high temperatures confirming local strain rates much higher than the nominal engineering ones after strain localization.

This work uses experimental data from A2-70 stainless steel specimens subjected to tension at different temperatures and strain rates, by motor-driven machine, hydraulic machine, and Split Hopkinson Tension Bar (SHTB). All tests are supported by camera acquisitions for measuring the effective evolving neck sections; the experimental true stress-true strain curves are then processed for deriving the flow curves then extracting and separating the functions of thermal softening and dynamic amplification.

\section{Experimental campaign and hardening model}

This work is based on an extensive experimental campaign on A2-70 stainless steel. Tensile tests on cylindrical specimens with nominal $3 \mathrm{~mm}$ diameter and $9 \mathrm{~mm}$ gauge length included static tests at different constant temperatures by motor-driven machine, dynamic tests at intermediate strain rates and different initial temperatures by hydraulic machine, and dynamic tests at high strain rates and initial room temperature by SHTB. In all tests, the evolving minimum diameter of the specimens was measured by image analysis of frames acquired by a standard high-resolution camera in the quasi-static tests and by a Phantom V711 speed camera in the intermediate and high-strain rate tests. Then, associating the diameter $d$ to the current load value $F$, it is possible to obtain the actual true stress $\sigma_{\text {True }}$, true strain $\varepsilon_{\text {True }}$ and true strain rate $\dot{\varepsilon}_{\text {True }}$ as shown in eqs. (1), (2) and (3).

$$
\begin{gathered}
\sigma_{\text {True }}=\frac{F}{\pi / 4 \cdot d^{2}} \\
\varepsilon_{\text {True }}=2 \cdot \operatorname{Ln}\left(\frac{d_{0}}{d}\right) \\
\dot{\varepsilon}_{\text {True }}=\frac{\partial \varepsilon}{\partial t}
\end{gathered}
$$

The experimental true stress-true strain curves (eqs. (1) and (2)) and the experimental strain rate histories (eqs. (2) and (3)) under the above different combinations of strain rates $\dot{\varepsilon}$ and temperatures $T$, were used for identifying the hardening function of the material affected at the 
same time by dynamic and softening effects, according to a general material model based on the multiplicative function shown in eq. (4).

$$
\sigma_{E q}(\varepsilon, \dot{\varepsilon}, T)=\sigma_{E q_{S t}}(\varepsilon) \cdot R(\varepsilon, \dot{\varepsilon}) \cdot\left[S_{S T}(\varepsilon, T)+S_{D Y N}(\varepsilon, \dot{\varepsilon}, T)\right]
$$

In this equation, $\sigma_{E q}$ is the flow stress incorporating dynamics and thermal effects, $\sigma_{E q_{S t}}$ is the reference flow stress (at static rate and room temperature), $R$ is the strain rate amplification and $S_{S T}+S_{D Y N}$ is the thermal softening, with the first term derived from static tests at constant temperature and the second term complementing the first one in case of dynamic tests with variable temperature. The coupling between strain rate and temperature effects is indirectly included in the dynamic thermal softening function because the temperature variability is generated and governed by the dynamics; then, the dynamic amplification only expresses the pure-rate effect.

The reference flow curve obviously depends on the strain alone and the static thermal softening was already demonstrated to include a remarkable dependence on the strain in previous works [16]: $\sigma_{E q_{S t}}$ and $S_{S T}$ are just recalled here for completing the identification of the other material constitutive functions, $R$ and $S_{D Y N}$.

The new tests carried out at dynamic rates and different initial temperatures, shown that $S_{S T}$ alone is not sufficient for describing the dynamic experiments and the new term $S_{D Y N}$ is necessary for modelling the difference between thermal softening at static and dynamic rates; this led to more realistic estimations of the dynamic amplifications $R$.

\subsection{Static tests at different constant temperatures}

The experimental true curves obtained from the static test at $20,40,80,140,200$ and $300{ }^{\circ} \mathrm{C}$ are shown in Fig. 1a as scatter plots. The corresponding necking strains $\varepsilon_{n}$ are reported in Table 1.
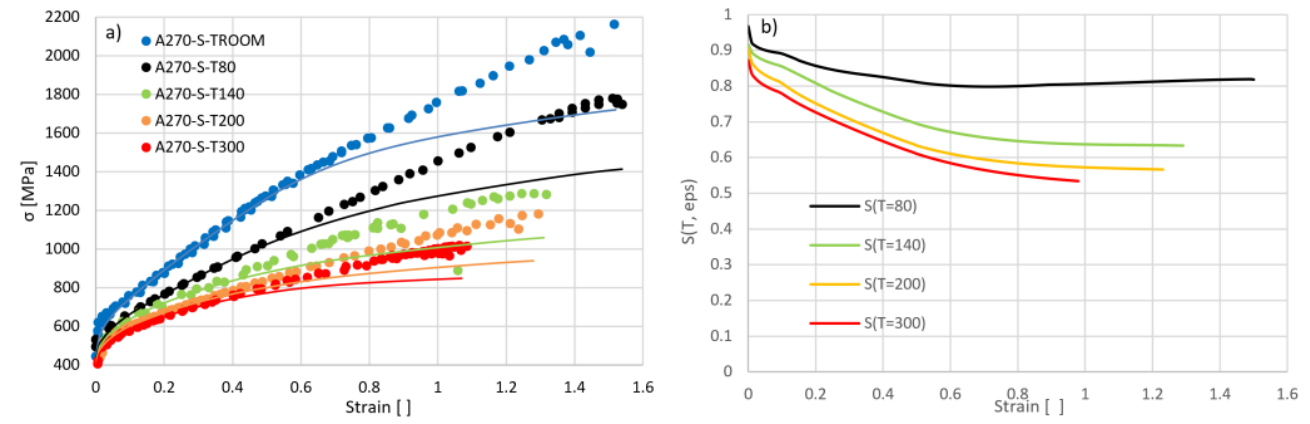

Fig. 1. Static tests: (a) True stress-strain experimental data at 20, 80, 140, 200 and $300{ }^{\circ} \mathrm{C}$ (scatter plots) and corresponding Mises curves (lines); (b) Static thermal softening functions $S_{S T}$.

It is very evident that, despite every test is conducted at a constant temperature, the obtained true curves are characterized by very different necking strains and overall curve shapes. As shown in [16], such evidence demonstrates that the temperature effect is coupled with the strain within the function $S_{S T}$, applying to isothermal static tests. Considering the equivalent curves obtained from these true curves via the MLR necking correction [4] (shown as solid lines in the same Fig. 1a), $S_{S T}$ is derived according to eq. (5).

$$
S_{S T}(\varepsilon, T)=\sigma_{E q}(\varepsilon, T) / \sigma_{E q_{S t}}(\varepsilon)
$$


The static thermal softening functions obtained from eq. (5) at fixed temperatures of 80 , 140,200 and $300{ }^{\circ} \mathrm{C}$ are shown in Fig. $1 \mathrm{~b}$, confirming that $S_{S T}$ at fixed temperatures is very variable with the strain. The two-variables function $S_{S T}(\varepsilon, T)$ expressing such coupled effect is taken from [16] where it was derived as a 3D best-fit of experimental data points from eq. (5).

Table 1. Necking strain $\varepsilon_{n}$ from static tensile tests at 20, $80,140,200$ and $300{ }^{\circ} \mathrm{C}$.

\begin{tabular}{|c|c|c|c|c|c|}
\hline & S-T20 & S-T80 & S-T140 & S-T200 & S-T300 \\
\hline$\varepsilon_{n}$ & 0.44 & 0.35 & 0.19 & 0.18 & 0.18 \\
\hline
\end{tabular}

\subsection{Dynamic tests at initial room temperature}

Dynamic tests at initial room temperature have been conducted by means of an SHTB setup at high strain rates (reference true strain rates 800 and $1800 \mathrm{~s}^{-1}$ ) and by a hydraulic machine at intermediate strain rates (reference true strain rates 1 and $10 \mathrm{~s}^{-1}$ ). The reference strain rate is the plateau value of the true strain rate history. In particular, the plateau extends from the end of the rise time to just before the necking onset.

The true curves obtained from these experiments are shown in Fig. 2a, compared to the static true curve at room temperature. A remarkable strain rate effect is evident throughout all the range between 1 and $1800 \mathrm{~s}^{-1}$ as higher strain rates deliver higher true curves, although, at large strains, the thermal softening promoted by the self-heating typical of fast plastic dissipation prevails on the dynamic amplification and makes the dynamic true curves lower than the static one.

The strain rate histories are shown in Fig. 2b, with Fig. 2c just magnifying the vertical axis for making visible the lower rate histories. All tests exhibit the typical surge of the strain rate beginning at the necking onset, already analysed by the authors in previous works [10][11][12][13] and by Zhang et al. [14] and Zhang et al. [15], leading to very large strain rates at failure, nearly ten times greater than the strain rate values at the plateau.

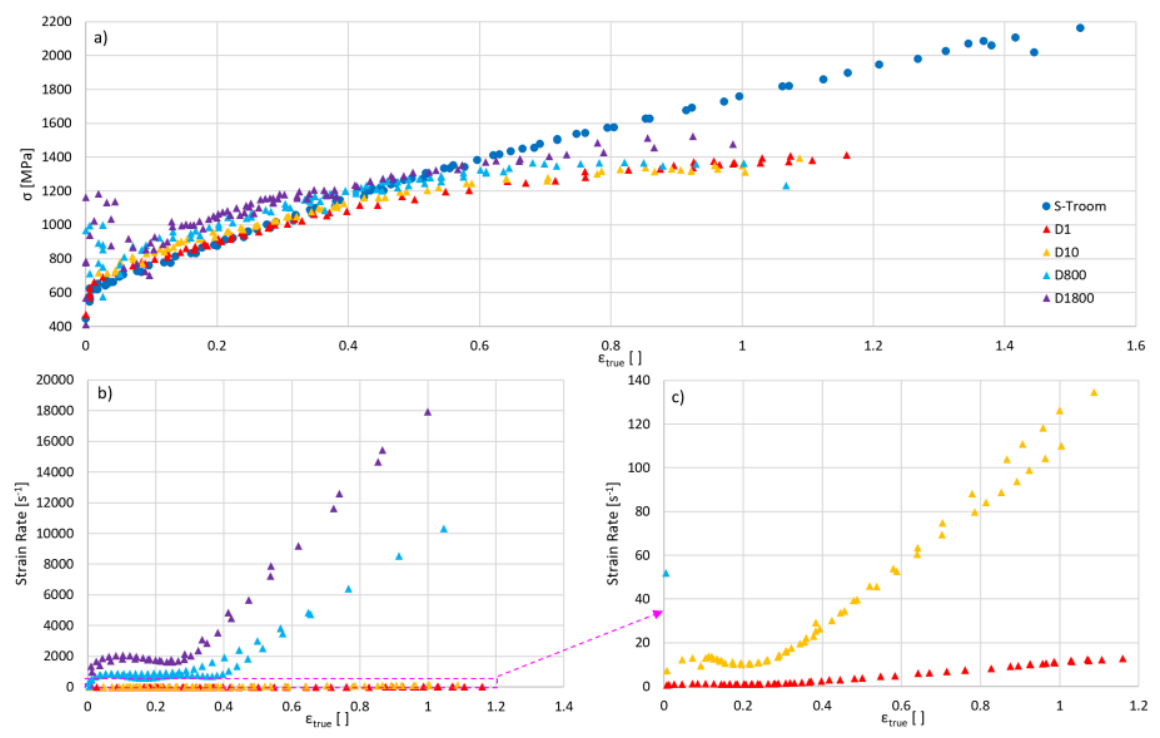

Fig. 2. Dynamic experiments at initial room temperature: (a) true stress-strain curves compared to the static curve at room temperature; (b) Strain rate vs strain curves and (c) magnification. 


\subsection{Dynamic tests at different initial temperatures}

In order to check whether or not the thermal softening at static rates, $S_{S T}$, was also suitable for describing the thermal softening at dynamic rates, additional tests were performed at the nominal strain rate of $10 \mathrm{~s}^{-1}$ imposing different initial temperatures $\left(20,60,100,150{ }^{\circ} \mathrm{C}\right)$. The obtained true curves are very different from each other, as shown in Fig. 3a, while the strain rate histories in Fig. $3 \mathrm{~b}$ are almost identical to each other, implying that also the dynamic amplification $R(\dot{\varepsilon})$ is almost the same for all tests. Therefore, the clear differences between the true curves can only be caused by the thermal softening which, then, can be assessed in detail.
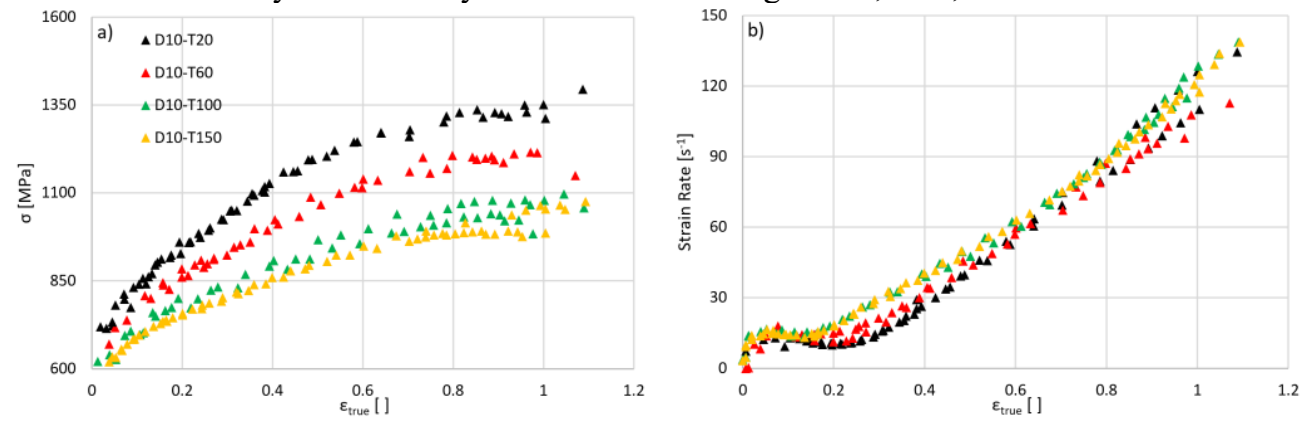

Fig. 3. Tests at the same strain rate $\left(10 \mathrm{~s}^{-1}\right)$ and different initial temperatures: (a) true stress-strain curves; (b) Strain rate-strain curves.

First, we try to check whether or not the static softening $S_{S T}$ alone is also representative of the thermal softening under dynamic conditions. If $S_{S T}$ is sufficient for modelling the thermal softening also at high strain rates, then $S_{D Y N}$ of eq. (4) is null and there is no coupling between strain rate and temperature; in such case, if we remove the effect of different initial temperatures from the experimental true curves of Fig. 3a, by way of the known function $S_{S T}$, we should obtain the same "cooled-down" flow curves from all tests of this series: in fact, if $S_{D Y N}$ does not exist, eq. (4) should yield to eq. (6), where $\dot{\varepsilon}_{10}$ is the strain rate history of Fig. $3 \mathrm{~b}, T_{20}, T_{60}, T_{100}$ and $T_{150}$ are the temperature histories of the dynamic tests at hand including the temperature increases due to the adiabatic self heating from fast plastic dissipation, and $\sigma_{E q}\left(\varepsilon, \dot{\varepsilon}_{10}, T_{i}\right)$ is the Mises curve of the test at nominal rate $10 \mathrm{~s}^{-1}$ and initial temperature $T_{i}$, obtained by the MLR necking correction of the respective true curves of Fig. 3a.

$$
\frac{\sigma_{E q}\left(\varepsilon, \dot{\varepsilon}_{10}, T_{20}\right)}{S_{S T}\left(\varepsilon, T_{20}\right)}=\frac{\sigma_{E q}\left(\varepsilon, \dot{\varepsilon}_{10}, T_{60}\right)}{S_{S T}\left(\varepsilon, T_{60}\right)}=\frac{\sigma_{E q}\left(\varepsilon, \dot{\varepsilon}_{10}, T_{100}\right)}{S_{S T}\left(\varepsilon, T_{100}\right)}=\frac{\sigma_{E q}\left(\varepsilon, \dot{\varepsilon}_{10}, T_{150}\right)}{S_{S T}\left(\varepsilon, T_{150}\right)}=\sigma_{E q_{S t}}(\varepsilon) \cdot R\left(\varepsilon, \dot{\varepsilon}_{10}\right)
$$

The knowledge of $\sigma_{E q}\left(\varepsilon, \dot{\varepsilon}_{10}, T_{i}\right)$ also allows to calculate the evolving temperature of the above tests by assuming a Taylor-Quinney coefficient $\mathrm{TQ}=1$, according to the incontrovertible evidence presented in [6].

The temperature histories calculated for the four tests at hand are reported in Fig. 4a, while the corresponding flow curves, virtually "cooled down" at room temperature by eq. (6), by way of $S_{S T}$ alone, are shown in Fig. $4 \mathrm{~b}$. It is evident that the flow curves cooled down with only $S_{S T}$ remarkably depart to each other at moderate strains. This means that $S_{S T}$ alone is not sufficient for the describing the thermal softening at dynamic rates and variable temperatures, because a coupling between temperature and dynamic effects exists through the term $\frac{\partial S}{\partial T} \cdot \frac{\partial T}{\partial \varepsilon}$ discussed in [16], where the "temperature slope" $\frac{\partial T}{\partial \varepsilon}$ is proportional to the strain rate. In fact, static rates obviously correspond to zero slope while higher rates induce steeper slopes for two reasons: the first is the progressive migration from isothermal to adiabatic conversion of plastic work; 
the second is that higher rates induce higher flow curves and, then, more plastic work dissipation and higher temperature slopes.

The curves from tests at initial temperatures of 100 and $150{ }^{\circ} \mathrm{C}$ are rather close to each other, indicating that $S_{D Y N}$ is going to vanish at initial temperatures in that range: we assume here that $S_{D Y N}$ is completely vanished at $150^{\circ} \mathrm{C}$.
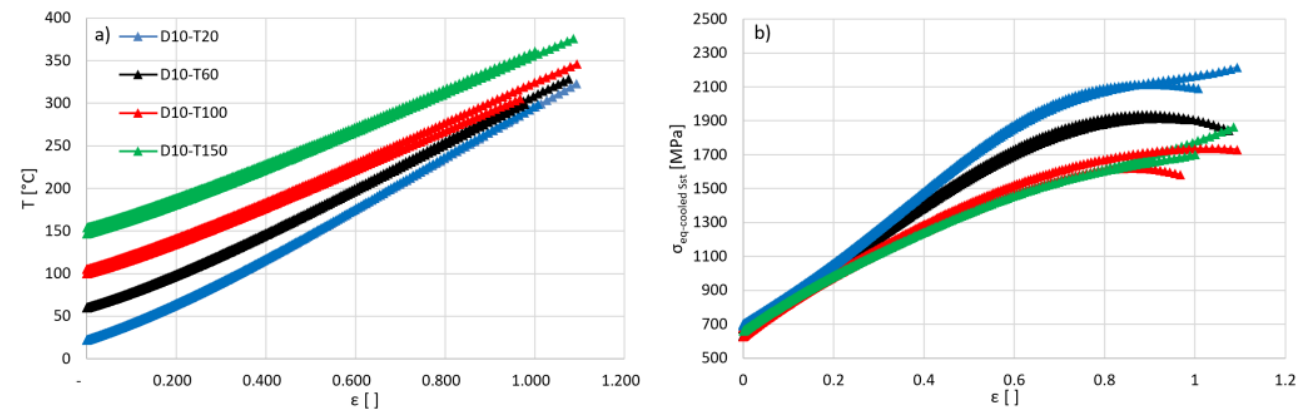

Fig. 4. Dynamic tests at the same strain rate $\left(10 \mathrm{~s}^{-1}\right)$ and different initial temperatures: (a) Temperature histories and (b) cooled equivalent curves considering only the static thermal softening $S_{S T}$.

Then, $S_{D Y N}$ can be evaluated without discerning its single terms but globally deriving it as the function capable of making the flow curves from the dynamic tests at initial $T_{20}, T_{60}, T_{100}$, cooled down by both thermal contribution $\left(S_{S T}+S_{D Y N}\right)$, equal to each other and equal to the flow curve of tests $T_{150}$ cooled down by $S_{S T}$ alone. According to eq. (4), this means that $S_{D Y N}$, for the test at $10 \mathrm{~s}^{-1}$ undergoing the temperature history $T_{i}$, can be derived from experimental data according to eq. (7).

$$
S_{D Y N}\left(\varepsilon, \dot{\varepsilon}_{10}, T_{i}\right)=\frac{\sigma_{E q}\left(\varepsilon, \dot{\varepsilon}_{10}, T_{i}\right)}{\sigma_{E q_{S t}}\left(\varepsilon, \dot{\varepsilon}_{10}, T_{150}\right)} \cdot S_{S T}\left(\varepsilon, T_{150}\right)-S_{S T}\left(\varepsilon, T_{i}\right)
$$

The functions $S_{D Y N}$ so obtained from the experiments are shown in Fig. 5a, where the curve for the $T_{100}$ temperature history is close to zero. The final cooled down curves considering the complete $S_{T O T}=S_{S T}+S_{D Y N}$ are shown in Fig. 5b. In this case, all the cooled down curves, expressing the static reference curves only amplified by the dynamic amplification $R\left(\dot{\varepsilon}_{10}\right)$, are identical to each other confirming the correctness of the procedure for determining $S_{D Y N}$.
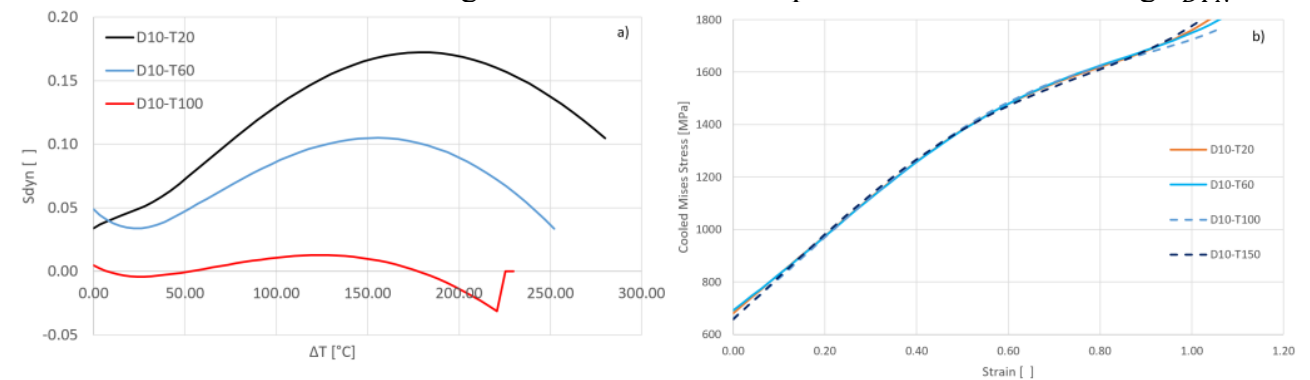

Fig. 5. Dynamic tests at the same strain rate $\left(10 \mathrm{~s}^{-1}\right)$ and different initial temperatures: (a) Dynamic softening; (b) Equivalent curves cooled down considering the total softening $S_{T O T}$.

The thermal softening functions derived so far are assumed to apply with whatever combination of strain rate and temperature of the experimental campaign. Other tests at fixed rate and different initial temperatures, e.g., $800 \mathrm{~s}^{-1}$ and $60,100,150{ }^{\circ} \mathrm{C}$, should be ran for verifying such assumption, although the derivation of $R$ in the next section may be of help for a preliminary assessment of its validity. 


\subsection{Strain rate amplification function $R$}

The knowledge of the total thermal softening $S_{S T}+S_{D Y N}$ allows to derive the dynamic amplification $R$ from the output of the dynamic tests. In fact, rearranging eq. (4), it is possible to obtain the function describing the strain rate effect from each test, as shown in eq. (6).

$$
R\left(\dot{\varepsilon}_{i}\right)=\frac{\sigma_{E q}\left(\varepsilon, \dot{\varepsilon}_{i}, T_{i}\right)}{\sigma_{E q_{S t}} \cdot\left[S_{S T}\left(\varepsilon, T_{i}\right)+S_{D Y N}\left(\dot{\varepsilon}_{i}, T_{i}\right)\right]}
$$

The strain rate amplification obtained as above from all dynamic tests is plotted against the plastic strain in Fig. 6a, while a magnification of the plot axes in Fig. $6 \mathrm{~b}$ evidences that no surge in the dynamic amplification occurs at the necking onset (black dots at strains between 0.21 and 0.27 ), despite a remarkable surge in the strain rate is known to occur at the same strain levels as already seen in Fig. 2b,c.
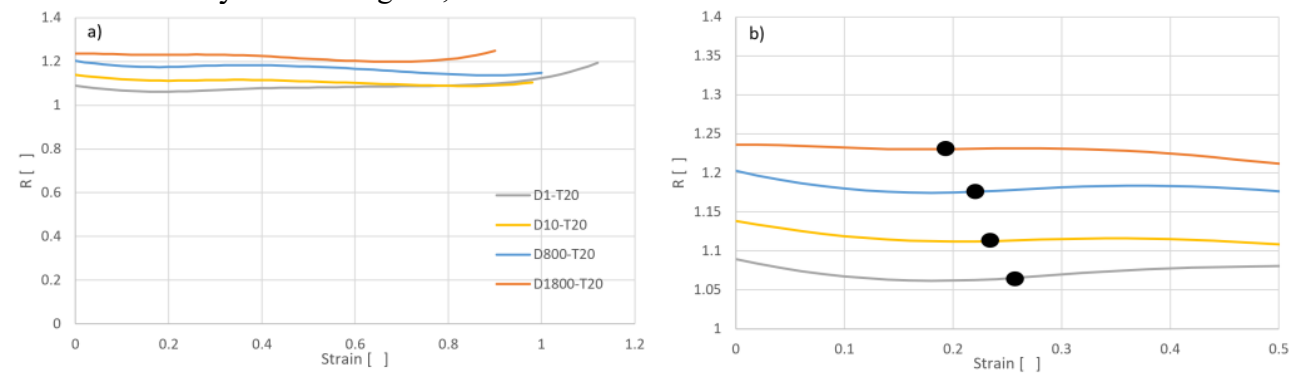

Fig. 6. Dynamic amplification function R vs strain for all the dynamic tests at room temperature (a) and magnification (b).

The strain rate sensitivity of the A2-70 steel is evident throughout the range from $1 \mathrm{~s}^{-1}$ to $1800 \mathrm{~s}^{-1}$ since the values of $R$ are greater/smaller according to the higher/lower values of the current strain rate. At the same time, the constancy of $R$ beyond the necking onset, evidenced above for all tests despite the corresponding strain rate surge, proves that the dynamic amplification quits to evolve at such strain level and remains locked at the current value, as already found by the authors in [10][11][12][13].

Such locking of the strain rate effect is better highlighted in Fig. 7 for the dynamic tests at $1 \mathrm{~s}^{-1}$ and $1800 \mathrm{~s}^{-1}$, representative of all dynamic tests ran in this work. In fact, before necking onset (vertical dashed lines at strains 0.27 and 0.21 ) the constancy of $R$ for each test is ensured and justified by the constancy of $\dot{\varepsilon}$ all over the plateau; but, after necking onset, the quick surge of $\dot{\varepsilon}$ (secondary axis on the right side of each plot in Fig. 7) while $R$ remains constant (primary axis on the left side of each plot) clearly demonstrates the above locking phenomenon.
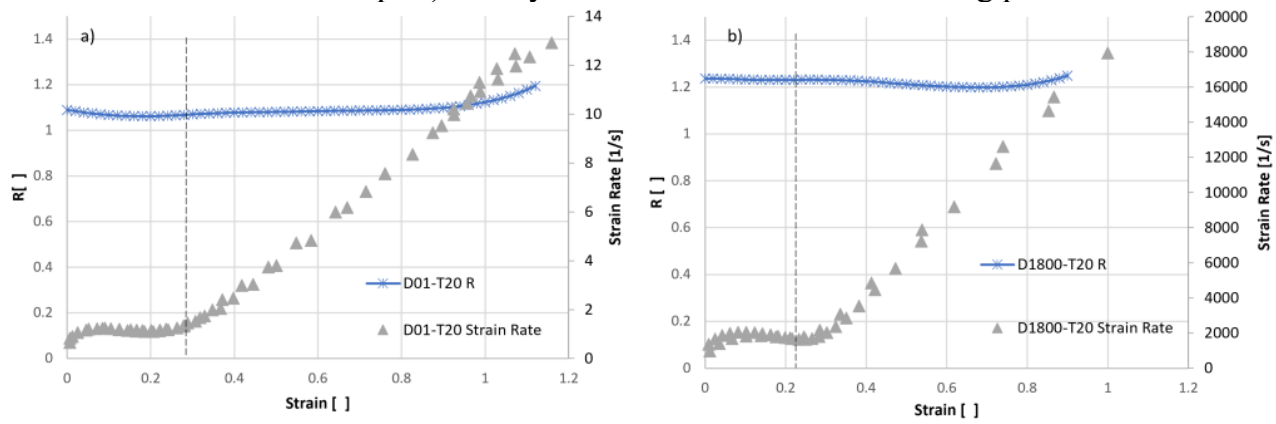

Fig. 7. True strain rate histories and dynamic amplification histories (double vertical axis) from dynamic tests at $1 \mathrm{~s}^{-1}$ (a) and $1800 \mathrm{~s}^{-1}(\mathrm{~b})$. 
It is important to highlight that the locking mechanism evidenced here does not mean that the material response is independent of the strain rate: it only means that the sensitivity of the material to the strain rate, remarkable and clearly visible in the Figures above, quits evolving and remains locked at the current value as the necking onset takes place.

If the strain rate at necking onset is low (tests at $1 \mathrm{~s}^{-1}$ ), $R \approx 1.06$. If, instead, the strain rate at necking onset is high (tests at $1800 \mathrm{~s}^{-1}$ ), $R \approx 1.22$. Then, the sensitivity of the A2-70 steel to the strain rate is remarkably high, as its dynamic amplification of the flow stress varies from $6 \%$ to $22 \%$ in the investigated dynamic range. These observations further support the previous findings of the authors in [12] for a different steel with very early necking onset and are also confirmed by Zhang et al. [14] for a titanium alloy.

Then, the function $R(\dot{\varepsilon})$ can be identified from experiments by only considering the prenecking phase, i.e., before such function is affected by the locking phenomena. Therefore, each test delivers only a point $\left(\dot{\varepsilon}_{i}, R_{i}\right)$ of the curve $R(\dot{\varepsilon})$. Each point is identified by the constant strain rate during the plateau and by the corresponding value of $R$ from eq. (6), constant as well.
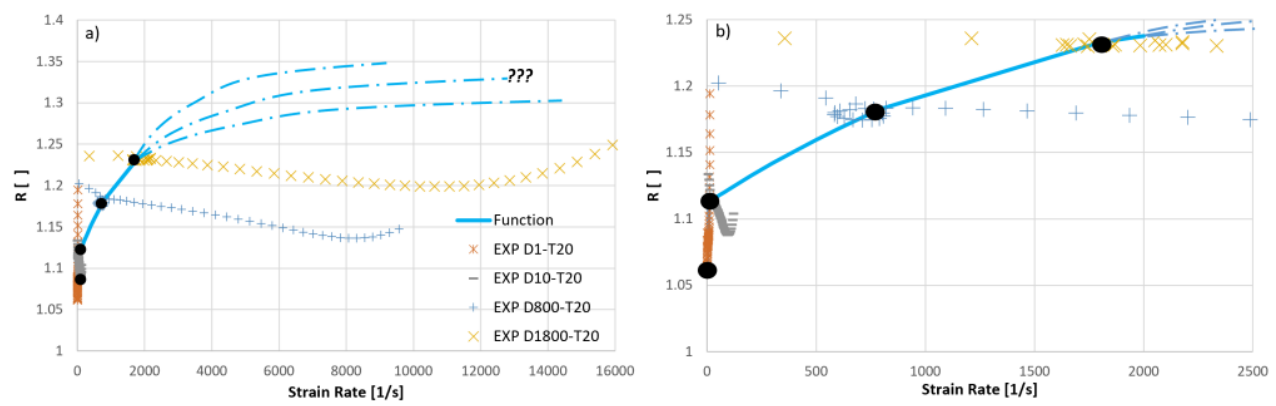

Fig. 8. Dynamic amplification versus strain rate at different magnification levels

Fig. 8 shows the function $R(\dot{\varepsilon})$ as a blue piecewise solid curve which interpolates the four black-dotted single points obtained from the dynamic tests as mentioned above, together with the four complete histories of the dynamic amplification acquired all over each test until failure, including the post-necking stages where $R$ is locked. The dash-dotted sketch curves prolonging the function $R(\dot{\varepsilon})$ beyond $1800 \mathrm{~s}^{-1}$ suggest three qualitative possible evolutions of the dynamic amplification: for identifying by experiments the real trend of $R(\dot{\varepsilon})$ at rates of $4^{\prime} 000,6^{\prime} 000$, $10^{\prime} 000$ or $20^{\prime} 000 \mathrm{~s}^{-1}$, such rates should be reached before the necking onset and before the locking takes place (i.e. at the plateau of the incident wave), which is quite impossible in standard-sized Hopkinson bar tests.

According to the above considerations, the effective function $R(\dot{\varepsilon})$ unaffected by the postnecking blockage is only known up to $1800 \mathrm{~s}^{-1}$, despite the complete $R$ histories including the locked post-necking range extend up to $16^{\prime} 000 \mathrm{~s}^{-1}, 9^{\prime} 000 \mathrm{~s}^{-1}, 120 \mathrm{~s}^{-1}$ and $12 \mathrm{~s}^{-1}$ respectively for the D1800, D800, D10 and D01 tests.

In Fig. 8b. the plot axes are magnified to slightly expand the histories of the D01 and D10 tests and to highlight that, for example, the purely-experimental dynamic amplification at 1800 $\mathrm{s}^{-1}$ assumes two completely different values depending on whether or not the necking and the consequent locking of the strain rate effect have already took place: in D800 tests the strain rate of $1800 \mathrm{~s}^{-1}$ is achieved after necking onset therefore the dynamic amplification is already "frozen" at a value around 1.18, instead in D1800 tests the strain rate of $1800 \mathrm{~s}^{-1}$ is achieved before necking onset therefore the function $R$ had the possibility of evolving slightly more, up to a value around 1.23, before being subjected to the locking and remaining constant until the specimen failure.

Similar considerations apply to whatever strain rate of a dynamic test: if the given value of $\dot{\varepsilon}$ refers to a pre-necking stage, then the dynamic amplification is delivered by the current value 
of the function $R=R(\dot{\varepsilon})$; if, instead, the given value of $\dot{\varepsilon}$ refers to a post-necking stage, then the current « locked» value of the dynamic amplification is delivered by a previous stage of the load history where the necking onset took place and may virtually span back to negligible rates, so that $1 \leq R_{\text {Locked }} \leq R(\dot{\varepsilon})$.

\section{Conclusions}

Tensile experiments at different strain rates and temperatures evidenced two important phenomena intrinsic in the elastoplastic response of a highly ductile late-necking A2-70 steel, namely the existence of a dynamic component of the thermal softening activated at high strain rates and the occurrence of the locking of the strain rate effect at necking onset. The latter phenomenon was already proved in previous works of the authors for a completely different, early necking steel. Both phenomena are derived here from experimental data and allowed to outline a new modelling approach suitable to properly take into account the combined and coupled effects of strain, strain rate and temperature on the elastoplastic response of the material. All the experiments were based on the acquisition of the current load and of the specimen minimum cross section through optical diameter measurements by a fast camera. Then, the effective current values of true stress-true strain-true strain rate were measured at different instants during the test. The reference flow curve at static rate and room temperature for the A2-70 steel was taken from previous works of the authors, together with the thermal softening at static rates, $S_{S T}$, incorporating a coupled dependence of the material response on temperature and strain.

The experiments performed here at fixed moderately high strain rate and different temperatures allowed to evidence and identify another contribution to the thermal softening, activated at dynamic strain rates and identified here as $S_{D Y N}$, filling the gap of the $S_{S T}$ function which, alone, was not sufficient to describe the thermal softening occurring in such dynamic experiments.

Then, the dynamic amplification $R$, expressing the sensitivity of the steel to high strain rates, was derived from dynamic tests at moderate (1-10 s $\left.\mathrm{s}^{-1}\right)$ and high strain rates (800 and 1800 $\mathrm{s}^{-1}$ ) at initial room temperature, accounting for the temperature increment due to plastic dissipation at fast rates. The simple ratio between dynamic and static flow stresses depurated of the above thermal effects delivered the histories of $R$ all over each test. The function $R(\dot{\varepsilon})$ resulting from the different dynamic tests shown a remarkable sensitivity of the A2-70 steel to the strain rates, as the dynamic amplifications varied from around $6 \%$ to $22 \%$ for strain rates ranging from $1 \mathrm{~s}^{-1}$ to $1800 \mathrm{~s}^{-1}$, provided that such rates were achieved before necking onset. At the same time, during each test, $R$ was found to remain constant after necking onset despite, all over such test phase, the strain rate underwent the typical steep surge due to the strain localization. Such evidence confirmed the locking of the strain rate effect at the necking onset previously evidenced by the authors for another steel; it also highlighted that the relevant data for characterizing $R(\dot{\varepsilon})$ by experiments can only be drawn before necking, limiting the amount of available data from each test to the plateau phase, where both $\dot{\varepsilon}$ and $R$ are constant. Therefore, due to the locking phenomena, a single dynamic test cannot deliver a segment of the curve $R(\dot{\varepsilon})$ despite the variability of the strain rate in the post-necking phase, while it can just deliver a single point $(\dot{\varepsilon}, R)$ of the dynamic amplification curve.

The rather general multiplicative material model proposed for enclosing such features is based the product of three functions expressing the reference flow stress, the dynamic amplification and the thermal softening. The latter function incorporates two additive contributions, respectively referring to static and dynamic rates. The locking phenomena, capping the dynamic amplification at necking onset depending on each given prescribed strain rate history, needs to be implemented as a further feature of the material model and will be the subject of successive developments of the present work. 


\section{References}

[1] L. Peroni, M. Scapin, C. Fichera, An advanced identification procedure for material model parameters based on image analysis, 10th European LS-DYNA Conference 2015, Würzburg, Germany (2015).

[2] M. Sasso, M. Fardmoshiri, E. Mancini, M. Rossi, L. Cortese. High speed imaging for material parameters calibration at high strain rate. The European Physical Journal Special Topics, 225(2), 295-309 (2016)

[3] P. W. Bridgman, Studies in large plastic flow and fracture (Vol. 177). New York: McGrawHill (1952)

[4] G. Mirone, Approximate model of the necking behaviour and application to the void growth prediction, International Journal of Damage Mechanics, 13(3), 241-261 (2004)

[5] G. Mirone, P. Verleysen, R. Barbagallo, Tensile testing of metals: Relationship between macroscopic engineering data and hardening variables at the semi-local scale, International Journal of Mechanical Sciences, 150, 154-167 (2019)

[6] R. Kapoor, S. Nemat-Nasser, Determination of temperature rise during high strain rate deformation. Mechanics of materials, 27(1), 1-12 (1998)

[7] S. M. Walley, W. G. Proud, P. J. Rae, J. E. Field, Comparison of two methods of measuring the rapid temperature rises in split Hopkinson bar specimens, Review of scientific instruments, 71(4), 1766-1771 (2000)

[8] C. Jovic, D. Wagner, P. Herve, G. Gary, L. Lazzarotto, Mechanical behaviour and temperature measurement during dynamic deformation on split Hopkinson bar of 304L stainless steel and 5754 aluminium alloy. In Journal de Physique IV (Proceedings) (Vol. 134, pp. 1279-1285). EDP sciences (2006)

[9] D. Rittel, L. H. Zhang, S. Osovski, The dependence of the Taylor-Quinney coefficient on the dynamic loading mode. Journal of the Mechanics and Physics of Solids, 107, 96-114 (2017)

[10] G. Mirone, R. Barbagallo, D. Corallo, S. Di Bella, Static and dynamic response of titanium alloy produced by electron beam melting. Procedia Structural Integrity, 2, 2355-2366 (2016)

[11] G. Mirone, D. Corallo, R. Barbagallo, Interaction of strain rate and necking on the stress-strain response of uniaxial tension tests by Hopkinson bar, Procedia Structural Integrity, 2, 974-985 (2016)

[12] G. Mirone, R. Barbagallo, F. Giudice, Locking of the strain rate effect in Hopkinson bar testing of a mild steel. International Journal of Impact Engineering, 130, 97-112 (2019)

[13] G. Mirone, R. Barbagallo, F. Giudice, S. Di Bella, Analysis and modelling of tensile and torsional behaviour at different strain rates of Ti6Al4V alloy additive manufactured by electron beam melting (EBM). Materials Science and Engineering: A, 793, 139916 (2020)

[14] L. Zhang, A. Pellegrino, D. Townsend, N. Petrinic, Strain rate and temperature dependent strain localization of a near $\alpha$ titanium alloy, International Journal of Impact Engineering, 145, 103676 (2020)

[15] L. H. Zhang, A. Pellegrino, N. Petrinic, Dynamic necking of a near $\alpha$ titanium alloy at high strain rates: Experiments and modelling. Defence Technology (2020)

[16] G. Mirone, R. Barbagallo, How sensitivity of metals to strain, strain rate and temperature affects necking onset and hardening in dynamic tests. International Journal of Mechanical Sciences, 195, 106249 (2021) 